NASA Technical Memorandum 89427

\title{
Estimability and Regulability of Linear Systems
}

\section{Yoram Baram and Thomas Kailath}

(NASA-TH-89427) ESTIMABILITY ANL
BEGULABIITY CF IINEAE SYSIEES (NASA) 31 p

N87-20790

Unclas

G3/66 45395

February 1987

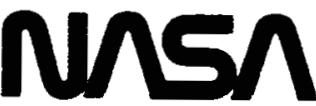

National Aeronautics and

Space Administration 


\section{Estimability and Regulability of Linear Systems}

Yoram Baram, Ames Research Center, Moffett Field, California

Thomas Kailath, Department of Engineering, Stanford University, Stanford, California

February 1987

\section{N/Sก}

National Aeronautics and

Space Administration

\section{Ames Research Center}

Moffett Field, California 94035 


\title{
ESTIMABILITY AND REGULABILITY OF LINEAR SYSTEMS \\ Yoram Baram* and Thomas Kailath**
}

\begin{abstract}
A linear state-space system will be said to be estimable if in estimating its state from its output the posterior error covariance matrix is strictly smaller than the prior covariance matrix. It will be said to be regulable if the quadratic cost of state feedback control is strictly smaller than the cost when no feedback is used. These properties, which are shown to be dual, are different from the well known observability and controllability properties of linear systems. Necessary and sufficient conditions for estimability and regulability are derived for time variant and time invariant systems, in discrete and continuous time.
\end{abstract}

*Y. Baram is with the NASA Ames Research Center, Moffett Field, CA 94035, on sabbatical leave from the Department of Electrical Engineering, Technion-Israel Institute of Technology, Haifa 32000 Israel.

**T. Kailath is with the Information Systems Laboratory, Department of Electrical Engineering, Stanford University, Stanford, CA 94305. His work was supported in part by the National Science Foundation under Grant DCI-84-21315-A1. 


\section{Introduction}

The benefit of using observation or feedback signals in state estimation or regulation of dynamical systems is normally manifested by the reduction of certain cost functions with respect to their values when no such signals are used. In this paper we introduce those properties of linear systems that yield mean-square error reduction in state estimation and quadratic cost reduction in state feedback control. These properties, which we call estimability and regulability, are different from the properties of observability and controllability, which were introduced by Kalman [1] and are widely recognized as key structural properties in linear estimation and control.

We shall say that a stochastic linear system is estimable if, in estimating its state from its output, the posterior error covariance matrix is strictly smaller than the prior state covariance matrix. This condition is equivalent to the condition that no direction in the state space at any time is orthogonal to all the past observations. It is also shown to be equivalent to the nonsingularity of a certain Gramian matrix. The notion of estimability is independent of that of observability in the sense that one does not imply the other. When a system is not estimable, the corresponding Kalman filter can be reduced to a lower-order estimator of the state process. A linear state-space system driven by white noise is shown to be a minimal order realization of its output process if and only if it is observable and estimable. These results apply to time-variant and time-invariant linear systems in discrete and continuous time. In the stationary case, the estimability condition leads directly to conditions derived by Baram and Shaked [2], [3], for minimality of the Kalman filter. 
We shall say that a linear system is regulable if, for any nonzero initial condition, the quadratic cost in applying optimal control is strictly smaller than the cost when no control is applied. This means that when a system is not regulable, the feedback signal may be eliminated for some nonzero initial condition without increasing the cost. Necessary and sufficient conditions for regulability are obtained for time-variant and time-invariant systems in discrete and continuous time. As might be expected, regulability and estimability are shown to be dual properties.

\section{Estimability}

Consider the system

$$
\begin{array}{rlrl}
x_{k+1} & =F_{k} x_{k}+G_{k} w_{k} & x_{k} \in R^{n} \\
y_{k} & =H_{k} x_{k}+v_{k} & k & =0,1,2, \ldots
\end{array}
$$

where $E\left\{x_{0}\right\}=0, E\left\{x_{0} x_{0}^{*}\right\}=\Pi_{0},\left\{w_{k}\right\}$ and $\left\{v_{k}\right\}$ are zero-mean sequences with $E\left\{x_{0} w_{k}^{*}\right\}=0, E\left\{w_{k} v_{j}^{*}\right\}=0, E\left\{w_{k} w_{j}^{*}\right\}=Q_{k} \delta_{k, j}, E\left\{v_{k} v_{j}^{*}\right\}=R_{k} \delta_{k, j}$. Also, $E[\cdot\}$ denotes expectation, * denotes Hermitian transpose, and $\delta_{k, j}$ denotes the Kronecker delta. Let $\hat{x}_{k}$ denote the linear least-squares estimate of $x_{k}$ given $\left[y_{k-1}, y_{k-2}, \ldots\right\}$ and let

$$
\Pi_{k}=E\left\{x_{k} x_{k}^{*}\right\} \text { and } P_{k}=E\left\{\left(x_{k}-\hat{x}_{k}\right)\left(x_{k}-\hat{x}_{k}\right)^{*}\right\}
$$


Definition 2.1. The system (2.1) is estimable if

$$
\begin{aligned}
& \qquad P_{k}<\Pi_{k} \text { for all } k \geq n \\
& \text { i.e., if and only if for any } g \in R^{n}, g \neq 0 \\
& \qquad{ }^{*} P_{k} g<g * \Pi_{k} g \text { for all } k \geq n
\end{aligned}
$$

or, in words, the posterior mean-square error in estimating any linear combination of the state variables is strictly smaller than the prior mean-square error. As will be shown later, the requirement $k \geq n$ is needed, since for $k<n$ the condition will immediately fail for, say, a scalar $\left\{y_{k}\right\}$ sequence.

The orthogonality property of the least-squares estimate implies that (2.2) will fail if and only if $g^{*} x_{k}$ is orthogonal to (uncorrelated with) the sequence $\left\{y_{j}, j \leq k\right\}$ for $k \geq n$. Conversely, (2.2) is equivalent to the condition

$$
\mathrm{g}^{*} \mathrm{E}\left\{\mathrm{x}_{\mathrm{k}} \mathrm{y}_{\mathrm{j}}^{*}\right\} \neq 0 \text { for some } \mathrm{j} \leq \mathrm{k}-1 \text { and all } \mathrm{k} \geq \mathrm{n} \text {. }
$$

Estimability means, by (2.2), that in estimating any linear combination of the state variables, the posterior mean-square error is strictly smaller than the prior mean-square error. Equivalently, by (2.3), it means that no direction in the state space for $k \geq n$ is orthogonal to all the past observations. 
Noting that

$$
x_{k}=\Phi_{k, j} x_{j}+\sum_{i=0}^{k-j} \Phi_{k, j+i} G_{j+i-1} w_{j+i-1}
$$

where $\Phi_{k, j}=F_{k-1} F_{k-2} \cdots F_{j}, \Phi_{k, k}=I$ denotes the system's state transition matrix, the estimability condition (2.3) can be written as

$$
g^{*} \Phi_{k, j} \mathbf{N}_{j} \neq 0
$$

for some $\mathrm{j} \leq \mathrm{k}-1$, where

$$
N_{j}=\Pi_{j} H_{j}
$$

Condition (2.4) holds if and only if the matrix sequence $\left\{\Phi_{k, j} N_{j}, j \leq k-1\right\}$ has column rank $n$ for all $k \geq n$, or, equivalently, the estimability Gramian

$$
w_{k}=\sum_{j=k-1}^{0} \Phi_{k, j} N_{j}^{N_{j}^{*} \Phi_{k, j}^{*}}
$$

has a full rank for all $k \geq n$. It can now be seen that we require $k \geq n$ in the definition of estimability since for a scalar $\left[y_{k}\right\}$ sequence the matrix sequence $\left\{\Phi_{k, j} N_{j}, j \leq k-1\right\}$ cannot have column rank $n$ for any $k<n$ and the condition will immediately fail. We note that the triple $\left\{H_{k}, \Phi_{k, j}, N_{j}\right.$, $j \leq k-1\}$ defines the covariance function of $\left\{y_{k}\right\}$

$$
R_{y}(k, j)=E\left\{y_{k} y_{j}^{*}\right\}=\left(R_{k}+H_{k} \Pi_{k} H_{k}^{*}\right) \delta_{k, j}+H_{k} \Phi_{k, j} N_{j} 1(k-j)+N_{k, j}^{*} \Phi_{k, j}^{*} 1(j-k)
$$


where $1(k-j)=1$ for $j<k, 0$ otherwise, and $\Pi_{k}$ satisfies the Lyapunov equation

$$
\Pi_{k+1}=F_{k} \Pi_{k} F_{k}^{*}+G_{k} Q_{k} G_{k}^{*}
$$

We have, then, the following result.

Theorem 2.1. The system (2.1) is estimable if and only if the following condition holds

$$
\operatorname{rank} W_{k}=n \text { for all } k \geq n
$$

We note that when the system is not estimable, the state estimate can be reduced, without loss of information, to a lower dimensional vector, which, in turn, results in the reduction of the Kalman filter to a lower dimensional linear estimator of the state.

\section{$\underline{\text { Remarks }}$}

1. The concept of estimability, which means that no direction in the state space is orthogonal to all the past observations, is an extension to the stochastic case of the concept of observability, which, in the deterministic case, means that the state can be deduced from the past observations. We note that this notion of "stochastic observability" is different from the one defined by Chen [4] as convergence of the error covariance matrix in estimating the initial state from a finite number of observations, when the prior covariance matrix is $\alpha I$ and $\alpha$ tends to infinity. While Chen's definition reduces to that of observability when the system is deterministic, the 
estimability notion is independent of that of observability, in the sense that, as can be readily verified by example, one does not imply the other.

2. Motivated by (2.3), let us examine the condition that no direction in the state space is orthogonal to all the past inputs (rather than outputs, as in (2.3)), i.e., that for any $g \in \mathbf{R}^{n}, g \neq 0$

$$
g^{*} E\left\{x_{k} w_{j}^{*}\right\} \neq 0 \text { for some } j \leq k-1 \text { for all } k \geq n
$$

which, noting that

$$
x_{k}=\Phi_{k, 0} x_{0}+\sum_{j=0}^{k-1} \Phi_{k, j+1} G_{j} \omega_{j}
$$

becomes

$$
{ }^{*} \Phi_{k, j+1} G_{j} Q_{j} \neq 0 \text { for some } j \leq k-1 \text {, for all } k \geq n
$$

for any $g \in \mathbf{R}^{n}, g \neq 0$. But the latter condition holds if and only if the matrix sequence $\left\{\Phi_{k, j} G_{j} Q_{j}, j \leq k-1\right\}$ has full column rank, which is the condition for reachability of the pair $\left(F_{j}, G_{j} Q_{j}\right)$. This condition, which has been termed by Bryson [5] "disturbability," is identical to the deterministic reachability condition when the input noise covariance $Q_{j}$ is nonsingular. This stands in contrast to the fact that estimability and observability are unrelated concepts. We also note that disturbability is not dual to estimability in the common system-theoretic sense. Such dual property will be presented in section 3 . 


\section{The Stationary Case}

Some useful equivalent forms of Theorem 2.1 can be obtained in the special case where the parameters $\{F, G, H, Q, R\}$ of the system (2.1) are time invariant. When the eigenvalues of $F$ are strictly inside the unit circle, the matrix $\pi_{k}=E\left\{x_{k} x_{k}^{*}\right\}$ has a steady-state value $\Pi$, which is the unique nonnegative solution of the equation (see, e.g., [6, p. 70])

$$
\Pi=F \Pi F^{*}+G Q G^{*}
$$

if $\Pi_{0}=\Pi$, then $\Pi_{k}$ has a constant value $\Pi$ for all $k \geq 0$ and the processes $x_{k}$ and $y_{k}$ are stationary. The matrix $N_{k}=\Pi_{k} H_{k}^{*}$ then has a constant value $\mathbf{N}=\pi^{*}$. The stationary system is estimable if the condition

$$
\mathrm{P}_{\mathrm{k}}<\pi \text { for all } \mathrm{k} \geq \mathrm{n}
$$

is satisfied. The following result is a direct consequence of Theorem 2.1 .

Corollary 2.1. The stationary $\operatorname{system}\left(F, G, H, Q, R, \Pi_{0}=\pi\right)$ is estimable if and only if the pair ( $F, F N)$ is reachable, i.e., $\operatorname{rank}\left[F N: F^{2} N: \cdots: F^{n} N\right]=n$

The Popov-Belevitch-Hautus test (see, e.g., [7], p. 135) can be used to obtain an equivalent characterization of estimability. Let us denote by $f_{i}^{*}$ a left eigenvector of $F$, corresponding to an eigenvalue $\lambda_{i}$, then $f_{i}^{*} A=\lambda_{i} f_{i}^{*}$. Multiplying (2.8) on the right by $f_{i}$, we obtain

$$
\pi f_{i}=\left(I-\lambda_{i} F\right)^{-1} G Q G^{*} f_{i}
$$


yielding

$$
N * f_{i}=H\left(I-\lambda_{i} F\right)^{-1} G Q G * f_{i}
$$

By the PBH test, the pair $(F, N)$ is reachable if and only if $N^{*} f_{i} \neq 0$ for any $f_{i}$ corresponding to each $\lambda_{i}$. Noting that $\lambda_{i} \neq 0$, we obtain, by (2.9) and Corollary 2.1, the following result, which relates the estimability property to the system's eigenstructure.

Corollary 2.2. The stationary system $\left(F, G, H, Q, R, \Pi_{0}=\Pi\right.$ ) is estimable if and only if for any left eigenvector $f_{i}^{*}$ corresponding to each eigenvalue $\lambda_{i}$ of $F$,

$$
H\left(\lambda_{i}^{-1} I-F\right)^{-1} G Q G * f_{i} \neq 0
$$

We note that condition $(2.10)$ is the same as the condition presented by Baram and Shaked [2] for minimality of the stationary Kalman filter associated with the system, in the sense that there is no linear estimator of its state, having order smaller than $n$. The above derivation is simpler than the one in [2], which would also be hard to generalize to the time-varying case. 
Now consider the system

$$
\begin{array}{ll}
\dot{x}(t)=F(t) x(t)+G(t) w(t) & x(t) \in \mathbb{R}^{n} \\
y(t)=H(t) x(t)+v(t) & t \geq 0
\end{array}
$$

where $E\{x(0)\}=0, E\left\{x(0) x^{*}(0)\right\}=\Pi(0),\{w(t)\}$ and $\{v(t)\}$ are zero-mean processes with $E\left\{x(0) w^{*}(t)\right\}=0, E\left\{w(t) w^{*}(s)\right\}=0, E\left[w(t) W^{*}(s)\right\}=Q(t) \delta(t-s)$ and $e\left\{v(t) v^{*}(s)\right\}=R(t) \delta(t-s)$. Let $\hat{x}(t)$ denote the linear least-squares estimate of $x(t)$ given $\{y(s), s \leq t\}$ and let

$$
\Pi(t)=E\left\{x(t) x^{*}(t)\right\} \text { and } P(t)=E\left\{[x(t)-\hat{x}(t)][x(t)-\hat{x}(t)]^{*}\right\}
$$

The system (2.11) is said to be estimable if the condition

$$
P(t)<\pi(t) \quad t>0
$$

is satisfied.

Let us denote by $\Phi(t, s)$ the system's transition matrix and let

$$
N(t)=\Pi(t) H^{*}(t)
$$

Defining the continuous time estimability Gramian by

$$
W(t)=\int_{t}^{0} \Phi(t, s) N(s) N^{*}(s) \Phi^{*}(t, s) d s
$$


and following the analysis of the discrete time case, we obtain the following result.

Theorem 2.2. The system (2.11) is estimable if and only if the following condition holds

$$
\text { rank } W(t)=n \text { for any } t>0
$$

In the time-invariant case, when the matrix $F$ has all its eigenvalues strictly in the left half plane, $\pi(t)$ reaches a steady-state value, which is the unique nonnegative solution of the equation

$$
F \Pi+\Pi F^{*}+G Q G^{*}=0
$$

When $n(0)=\pi$, the matrix $N(t)$ has a constant value $N=\pi H^{*}$ and the processes $\{x(t)\}$ and $\{y(t)\}$ are stationary. The stationary system is estimable if the condition

$$
P(t)<\pi \quad t>0
$$

holds. The following result is a direct consequence of Theorem 2.2.

Corollary 2.3. The stationary system (F, G, H, Q, R, $\Pi(0)=\Pi$ ) is estimable if and only if the pair $(F, N)$ is controllable.

Let us denote by $f_{1}^{*}$ a left eigenvector of $F$ corresponding to an eigenvalue $\lambda_{i}$. Multiplying $(2.13)$ on the right by $f_{i}$, we obtain 


$$
\pi f_{i}=\left(-\lambda_{i} I-E\right)^{-1} G Q G * f_{i}
$$

yielding

$$
N^{*} f_{i}=H^{*}\left(-\lambda_{i} I-F\right)^{-1} G Q G^{*} f_{i}
$$

Since, by the PBH test, the pair $(F, N)$ is controllable if and only if $\mathrm{N}^{*} \mathrm{f}_{\mathrm{i}} \neq 0$, Corollary 2.3 and equation (2.14) yield the following result.

Corollary 2.4. The stationary system ( $F, G, H, Q, R, \Pi(0)=\pi$ ) is estimable if and only if for any left eigenvector $f_{i}^{*}$ corresponding to each eigenvalue $\lambda_{i}$ of $F$,

$$
H\left(-\lambda_{i} I-F\right)^{-1} G Q G^{*} f_{i} \neq 0
$$

As in the discrete-time case, this is also the condition for minimality of the continuous-time stationary Kalman filter [3]. The above derivation is simpler than the one in [3] and the condition is shown to be a restriction to the stationary case of the more general estimability property.

\section{Minimal Realization of the Observation Process}

System estimability implies, by definition, that the observations contain information on all the state variables. It might be expected, then, that the minimality of any realization of the observation process would be strongly linked to the estimability property. The fact that estimability depends on the sequence $\left[\mathrm{N}_{k}\right\}$, which defines the covariance function of the output process, is a further indication of such a linkage. We first note that if we 
define the order of a state-space realization of a possibly nonstationary process as the dimension of its state process, then the order will be generally time-dependent. We shall say that a realization of such a process is minimal if its order is not greater than that of any other realization of the process at any time.

It is known (see, e.g., [8]) that the minimal order of any realization of a stationary process $\left\{\mathrm{y}_{\mathrm{k}}\right\}$, possessing a finite dimensional linear representation is the dimension of the Hilbert space $Y_{k}$ generated by $\left\{\hat{y}_{k+j}, j \geq 0\right\}$, where $\hat{y}_{k+j}$ denotes the least-squares estimate of $y_{k+j}$ given $\left\{y_{k-1}, y_{k-2}, \ldots, y_{0}\right\}$. To see that this is also true when $\left\{y_{k}\right\}$ is nonstationary, let $z_{k}$ denote a base of $Y_{k}$, i.e., a vector of minimal dimension from which any element of $Y_{k}$ can be obtained by a linear transformation. Since $z_{k+1} \in Y_{k+1} \subset Y_{k} \otimes v_{k}$, where $v_{k}=y_{k}-\hat{y}_{k}$ and $\otimes$ denotes the cartesian product and since $v_{k} \perp Y_{k}$, there exist matrices $A_{k}, B_{k}$, and $C_{k}$, such that $z_{k+1}=A_{k} z_{k}+B_{k} v_{k}$ and $y_{k}=C_{k} z_{k}+v_{k}$. By definition, $\operatorname{dim} z_{k}=\operatorname{dim} Y_{k}$. Suppose that there exists a realization of $\left\{y_{k}\right\}$, given by $q_{k+1}=\alpha_{k} q_{k}+\beta_{k} r_{k}, y_{k}=r_{k} q_{k}+t_{k}$, where $\left\{r_{k}\right\}$ is a white noise process, uncorrelated with $q_{0}$ and $\left\{t_{k}\right\}$ is a white noise process, uncorrelated with $\left\{r_{k}\right\}$, such that $\operatorname{dim} q_{k}<\operatorname{dim} Y_{k}$. Then we have $\left[\hat{y}_{k}, \hat{y}_{k+1}, \ldots\right]=\left[\gamma_{k}, \gamma_{k+1} \theta_{k+1, k}, \ldots\right] \hat{q}_{k}$ where $\theta_{k+j, k}$ is the transition matrix corresponding to $\alpha_{k}$ and $\hat{q}_{k}$ is the least squares estimate of $q_{k}$, given $\left\{y_{k-1}, y_{k-2}, \ldots\right\}$. But since the dimension of $y_{k}$ is equal to the covariance rank of $\left[\hat{y}_{k}^{T}: \hat{y}_{k+1}^{T}: \cdots\right]$, we have $\operatorname{dim} Y_{k} \leq \operatorname{dim} q_{k}<\operatorname{dim} Y_{k}$, a contradiction. It follows that the minimal order of any realization of $\left\{y_{k}\right\}$ at time $k$ is equal to the dimension of $Y_{k}$. 
Since, by (2.1), we have $\left[\hat{y}_{k}, \hat{y}_{k+1}, \ldots\right]=\left[H_{k}, H_{k+1} \phi_{k+1, k}, \ldots\right] \hat{x}_{k}$ it follows that $\operatorname{dim} Y_{k}=n$ if and only if (2.1) is observable and rank $E\left\{\hat{x}_{k} \hat{x}_{k}^{*}\right\}=n$. Since the latter, for $k \geq n$, is the condition for estimability we have the following result.

Theorem 2.3. The system (2.1) is a minimal order realization of the process $\left\{y_{k}\right\}$ for $k \geq n$ if and only if it is observable and estimable.

Restricting Theorem 2.3 to the stationary case and employing corollary 2.1 , we obtain the following result.

Corollary 2.5. The system ( F, G, H, Q, $R, \Pi_{0}=\Pi$ ) is a minimal realization of a stationary output process $\left\{y_{k}\right\}$ if and only if the triplet $(H, F, N)$ is observable and reachable.

Theorem 2.3 and corollary 2.2 further imply that in the stationary case the system is a minimal realization of its output process if and only if the pair $(H, F)$ is observable and condition (2.10) holds. This result, which was first presented in [9] is now extended by Theorem 2.3 to nonstationary processes. We also note that for the stationary case, the above analysis implies that an observable system of order $n$ is estimable if and only if the denominator degree of its spectral density function is $2 n$. These results can be extended to the continuous time case. 


\section{Regulability}

Consider a system

$$
x_{k+1}=F_{k} x_{k}+G_{k} u_{k}
$$

where the input sequence $\left[u_{k}\right]$ is to be selected so as to minimize the quadratic cost function

$$
J\left[x_{0},\left\{u_{k}\right\}, k\right]=\sum_{k=0}^{K-1} x_{k+1}^{*} L_{k+1} x_{k+1}+u_{k}^{*} M_{k} u_{k}
$$

where $L_{k}$ is a nonnegative matrix and $M_{k}$ is a positive definite matrix.

Definition 3.1. The system (3.1) is regulable if for any initial condition $x_{0} \neq 0$

$$
\min _{\left\{u_{k}\right\}} J\left[x_{0},\left\{u_{k}\right\}, K\right]<J\left[x_{0},\left\{u_{k}=0\right\}, k\right] \text { for all } k \geq n
$$

Regulability means that for any initial condition the optimal cost is strictly smaller than the cost when no input is used.

We next derive a Gramian rank test for regulability. Substituting

$$
x_{k}=\Phi_{k, 0} x_{0}+\sum_{j=0}^{k-1} \Phi_{k, j+1} G_{j} u_{j}
$$

in (3.2), we obtain 


$$
\begin{aligned}
J\left[w_{0},\left\{u_{k}\right\}, K\right]= & \sum_{k=0}^{K-1}\left\{x_{0}^{*} \Phi_{k+1,0}^{L}{ }_{k+1} \Phi_{k+1,0^{x} 0}\right. \\
& +2 x_{0}^{*} \Phi_{k+1,0}^{*}{ }_{k+1} \sum_{j=0}^{k} \Phi_{k+1, j+1}^{G}{ }^{u} j \\
& \left.+\left(\sum_{j=0}^{k} \Phi_{k+1, j+1}^{G} u_{j}\right)^{*} L_{k+1}\left(\sum_{j=0}^{k} \Phi_{k+1, j+1}^{G} u_{j}\right)+u_{k}^{* M} u_{k}\right\}
\end{aligned}
$$

Let

$$
A_{k, j}=L_{k+1}^{1 / 2} \Phi_{k+1, j+1} G_{j}, \quad b_{k}=L_{k+1}^{1 / 2} \Phi_{k+1}, 0_{0}^{x_{0}}
$$

and

$$
\begin{aligned}
& \bar{A}=\left[\begin{array}{ccccc}
A_{0,0} & & & & \\
A_{1,0} & A_{1,1} & & 0 \\
A_{2,0} & A_{2,1} & A_{2,2} & & \\
\vdots & & & \cdot & \\
\dot{A_{K-1,0}} & A_{K-1,1} & \ldots & A_{K-1, K-1}
\end{array}\right] \quad \bar{B}=\left[\begin{array}{c}
b_{0} \\
b_{1} \\
\vdots \\
b_{K-1}
\end{array}\right] \\
& \bar{M}=\left[\begin{array}{lll}
M_{1} & & \\
& \cdot & 0 \\
0 & & M_{K-1}
\end{array}\right] \\
& \overline{\mathrm{u}}=\left[\begin{array}{c}
\mathrm{u}_{0} \\
\mathrm{u}_{1} \\
\dot{u_{\mathrm{K}}-1}
\end{array}\right]
\end{aligned}
$$


then it can be readily verified that

$$
\begin{aligned}
\Delta J & \equiv J\left[x_{0},\left\{u_{k}\right\}, K\right]-J\left[x_{0},\left\{u_{k}=0\right\}, K\right] \\
& =\bar{u}^{*}\left[\bar{A}^{*} \bar{A}+\bar{M}\right] \bar{u}+2 \bar{b}^{*} \bar{A} \bar{u}
\end{aligned}
$$

We would like to find a necessary and sufficient condition for regulability, i.e., a condition under which $\min _{\left\{u_{k}\right\}} \Delta J<0$. Obviously, we have $\underset{\left\{u_{k}\right\}}{\min } \Delta J \leq 0$ so, we would like to find a condition under which

$$
\min _{\left\{u_{k}\right\}} \Delta J \neq 0
$$

Differentiating (3.3) and equating to zero, we obtain

$$
\frac{\partial \Delta J}{\partial u}=2 \bar{u}^{*}\left(\bar{A}^{*} \bar{A}+\bar{M}\right)+2 \bar{b}^{*} \bar{A}=0
$$

Since $\bar{M}$ has full rank, so does $(\bar{A} * \bar{A}+\bar{M})$. Hence, the optimal input is

$$
u=-(\bar{A} * \bar{A}+\bar{M})^{-1} \bar{A}^{*} \bar{b}
$$

yielding

$$
\min _{\left\{u_{k}\right\}} \Delta J=-\bar{b} * \bar{A}\left(\bar{A}^{*} \bar{A}+\bar{M}\right)^{-1} \bar{A}^{*} \bar{b}
$$

It follows that (3.4) holds if and only if $\bar{b}$ is not in the null space of $\bar{A}^{*}$, i.e., if and only if 
The regulability condition is then

$$
\sum_{k=0}^{K-1}\left(\sum_{j=k}^{K-1} A_{j, k}^{*} b_{j}\right)^{*}\left(\sum_{j=k}^{K-1} A_{j, k_{j}^{*}}^{*} b_{j} \neq 0\right.
$$

or, for any $x_{0} \neq 0$

$$
x_{0}^{*} \sum_{k=0}^{K-1}\left(\sum_{j=k}^{K-1} \Phi_{j+1,0^{L}}^{L}{ }_{j+1}^{\Phi} j+1, k+1\right) G_{k} G_{k}^{*}\left(\sum_{j=k}^{K-1} \Phi_{j+1, k+1}^{*} L_{j+1}^{\Phi} j+1,0\right) x_{0} \neq 0
$$

Noting that $\Phi_{j+1,0}=\Phi_{j+1, k+1} \Phi_{k+1,0}$ the condition becomes

$$
x_{0}^{*} \sum_{k=0}^{K-1} \Phi_{k+1,0}^{*}\left(\sum_{j=k}^{K-1} \Phi_{j+1, k+1}^{*}{ }_{j+1}^{\Phi}{ }_{j+1, k+1}\right) G_{k} G_{k}^{*}
$$

$$
\times\left(\sum_{j=k}^{K-1} \Phi_{j+1, k+1}^{*} L_{j+1}^{\Phi} j+1, k+1\right) \Phi_{k+1,0^{x}} \neq 0
$$

for any $x_{0} \neq 0$. The matrix

$$
\psi_{k}=\sum_{j=k}^{K-1} \Phi_{j+1, k+1}^{*} L_{j+1}^{\Phi} j+1, k+1
$$

can be seen to satisfy the (time-reversed) Lyapunov equation

$$
\psi_{k}=F_{k+1}^{*} \psi_{k+1} F_{k+1}+L_{k}, \quad \psi_{K}=L_{K}
$$

The regulability condition can now be written as 


$$
x_{0}^{*} W_{K} x_{0} \neq 0 \text { for any } x_{0} \neq 0
$$

where $W_{K}$ is the regulability Gramian

$$
W_{K}=\sum_{k=0}^{K-1} \Phi_{k+1}^{*} S_{k} S_{k}^{*} \Phi_{k+1,0}
$$

with

$$
S_{k}=\psi_{k} G_{k}
$$

We have proved the following result.

Theorem 3.1. The system (3.1) is regulable if and only if

$$
\text { rank } w_{K}=n \text { for all } K \geq n
$$

Suppose that the regulability condition is not satisfied. Then for some initial condition $x_{0} \neq 0$ the cost will not be reduced by applying any input signal $\left[u_{k}\right\}$. This initial condition can be found by solving the equation

$$
\left(\sum_{k=0}^{K-1} \Phi_{k+1,0}^{*} S_{k} S_{k}^{*} \Phi_{k+1,0}\right) x_{0}=0
$$

When the system is initialized at this initial condition, any state feedback control signal may be eliminated without increasing the cost. We note that the latter condition is analogous to the orthogonality condition $\mathrm{g}^{*} E\left[\mathrm{x}_{\mathrm{k}} \mathrm{y}_{\mathrm{j}}^{*}\right\}=0, \mathrm{~J} \leq \mathrm{k}$ in the estimation problem. 


\section{Time-Invariant Systems}

Now consider the time invariant system

$$
\mathrm{x}_{\mathrm{k}+1}=\mathrm{Fx}_{\mathrm{k}}+\mathrm{Gu}_{\mathrm{k}}, \quad \mathrm{k} \geq 0
$$

where $F$ is assumed to have all its eigenvalues strictly inside the unit circle and the input $\left\{u_{k}\right\}$ is selected so as to minimize the cost function

$$
J\left[x_{0},\left\{u_{k}\right\}\right]=x_{K}^{*}\left[x_{K}+\sum_{k=0}^{K-1} x_{k}^{*} L x_{k}+u_{k}^{*} M u_{k}\right.
$$

For $K \rightarrow \infty$, the matrix $\psi_{k}$, defined by (3.6), has a steady state value $\psi_{k}=\psi, k \geq 0$, which is the unique non-negative solution of the equation

$$
\psi=F^{*} \psi F+L
$$

Taking $\bar{L}=\psi$, we also have $\psi_{k}=\psi, k \geq 0$. This situation is the analog of stationarity of the stochastic system studied in section 2 . Denoting $S=\psi G$, we obtain the following result as a direct consequence of Theorem 3.1 .

Corollary 3.1. The system (3.8) with $[=\psi$ is regulable if and only if the pair $\left(E^{*}, S\right)$ is reachable.

Let $f_{i}$ denote an eigenvector of $F$, corresponding to an eigenvalue $\lambda_{i}$. Multiplying (3.9) on the right by $f_{i}$ we obtain

$$
\psi f_{i}=\left(I-\lambda_{i} F^{*}\right)^{-1} L f_{i}
$$


yielding

$$
S^{*} f_{i}=G^{*}\left(\lambda_{i}^{-1} I-F^{*}\right) L f_{i}
$$

By the Popov-Belevitch-Hautus test the pair $\left(F^{*}, S\right)$ is reachable if and only if $S *_{i} \neq 0$ for any $f_{i}$ corresponding to each $\lambda_{i}$. Noting that $\lambda_{i} \neq 0$, we obtain the following result from corollary 3.1 and equation (3.10).

Corollary 3.2. The system (3.8) with $\bar{L}=\psi$ is regulable if and only if for any eigenvector $f_{i}$, corresponding to each eigenvalue $\lambda_{i}$ of $F$,

$$
G^{*}\left(\lambda_{i}^{-1} I-F^{*}\right) L f_{i} \neq 0
$$

It follows from (3.11) that a necessary condition for regulability is that $L f_{i} \neq 0$ for any $f_{i}$ corresponding to each $\lambda_{i}$, which is equivalent to reachability of the pair $\left(F^{*}, L^{*}\right)$. Given that the latter condition is satisfied, a sufficient condition for regulability is that none of the zeroes of the system (G, $\left.F^{*}, L\right)$ is a reciprocal of any of its poles.

\section{Continuous-Time Systems}

Consider that the system

$$
\dot{x}(t)=F(t) x(t)+G(t) u(t), \quad t \geq 0
$$

where $\{u(t)\}$ is selected so as to minimize the cost function

$$
J[x(0),\{u(t)\}, T]=x^{*}(T) L(T) x(T)+\int_{0}^{T}\left[x^{*}(t) L(t) x(t)+u^{*}(t) M(t) u(t)\right] d t
$$


where $L(t)$ is a non-negative definite matrix and $M(t)$ is a positive definite matrix. The system is said to be regulable if we have, for any initial condition $x(0)$,

$$
\min _{\{u(t)\}} J[x(0),\{u(t)\}, T]<J[x(0),\{u(t)=0\}, T] \text { for any } t \geq 0
$$

Let us define the continuous-time regulability Gramian

$$
W(t)=\int_{0}^{T} \Phi^{*}(t, 0) S(t) S^{*}(t) \Phi(t, 0) d t
$$

where

$$
S(t)=\psi(t) G(t)
$$

and $\psi(t)$ satisfies the equation

$$
-\dot{\psi}(t)=\psi(t) F(t)+F^{*}(t) \psi(t)+L(t), \quad \psi(T)=L(T)
$$

Then, by applying limiting arguments to the analysis of the discrete time case or by similar direct calculations (see also some relevant calculations in [10, App. B]), we obtain the following result.

Theorem 3.2. The system (3.12) is regulable if and only if

$$
\operatorname{rank} W(t)=n \text { for any } t>0
$$


For the time invariant system

$$
\dot{x}(t)=F x(t)+G u(t), \quad t \geq 0
$$

where $F$ is assumed to have all its eigenvalues strictly in the left half plane and

$$
J[x(0),\{u(t)\}]=x^{*}(T)\left[x(T)+\int_{0}^{T}\left[x^{*}(t) \operatorname{Lx}(t)+u^{*}(t) M u(t)\right] d t\right.
$$

where $L$ is a non-negative definite matrix and $M$ is a positive definite matrix, we define

$$
S=\psi G
$$

where $\psi$ satisfies the equation

$$
\psi F+F^{*} \psi+L=0
$$

and, following the analysis of the discrete-time case, we obtain the following result.

\section{Corollary 3.3}

The system (3.13) with $\bar{L}=\downarrow$ is regulable if and only if the pair $\left(F^{*}, S\right)$ is controllable, or, equivalently, for any eigenvector $f_{i}$ corresponding to each eigenvalue $\lambda_{i}$ of $F$, the condition 


$$
G^{*}\left(-\lambda_{i} I-F^{*}\right)^{-1} L_{i} \neq 0
$$

is satisfied.

4. Duality

It can be seen that the estimability condition (2.6) and the regulability condition (3.7), which involve the Lyapunov equations (2.5) and (3.6), respectively, are similar, modulo time reversal. Such similarity, which has also been shown to exist between the Riccati equations associated with the estimation and the regulation problems, has been termed "duality" in the literature [1]. We now show that the duality between the estimability and the regulability properties is consistent with the one between the Lyapunov and the Riccati equations corresponding to the estimation and the regulation problems and, employing this duality, obtain the regulability condition from the estimability condition.

Estimability in the discrete-time case was defined in section 2 by the condition

$$
P_{k}<\Pi_{k}
$$

where $P_{k}$ is known to satisfy the Riccati equation

$$
P_{k+1}=F_{k} P_{k} F_{k}^{*}+G_{k} Q_{k} G_{k}^{*}-F_{k} P_{k} H_{k}^{*}\left(H_{k} P_{k} H_{k}^{*}+R_{k}\right)^{-1} H_{k} P_{k} F_{k}^{*}
$$

initialized at $P_{0}=\Pi_{0}$, while $\pi_{k}$ is known to satisfy the Lyapunov equation

$$
\Pi_{k+1}=F_{k} \Pi_{k} F_{k}^{*}+G_{k} Q_{k} G_{k}^{*}
$$


In the discrete time regulation problem the minimal cost is known to be $[11$, p. 494]

$$
\min _{\left\{u_{k}\right\}} J\left[x_{0},\left[u_{k}\right\}, K\right]=x_{0}^{*} r_{0} x_{0}
$$

where $\Gamma_{0}$ satisfies the (time-reversed) Riccati equation

$$
\Gamma_{k}=F_{k+1}^{*} \Gamma_{k+1} F_{k+1}+L_{k+1}-F_{k+1}^{*} \Gamma_{k+1} G_{k+1}\left[M_{k+1}+G_{k+1}^{*} \Gamma_{k+1} G_{k+1}\right]^{-1} G_{k+1}^{*} \Gamma_{k+1} F_{k+1}
$$

with the terminal condition $r_{K}=L_{K}$. The cost for zero input is obtained from (3.1) and (3.2) as

$$
J\left[x_{0},\left\{u_{k}=0\right\}, K\right]=x_{0}^{*} \Psi_{0} x_{0}
$$

where $\psi_{0}$ satisfies the (time-reversed) Lyapunov equation

$$
\psi_{k}=F_{k+1}^{*} \psi_{k+1} F_{k+1}+L_{k+1}
$$

with the terminal condition $\psi_{K}=L_{K}$. It follows from (4.4) and (4.6) that the regulability condition (3.3) is equivalent to the condition

$$
r_{0}<\Psi_{0}
$$

It can be seen that the regulability condition (4.8) and equations (4.5) and (4.7) are dual, modulo time reversal, to the estimability condition (4.1) 
and equations (4.2) and (4.3). It follows that the regulability condition, dual to the estimability condition

$$
\operatorname{rank} \sum_{k=K-1}^{0} \Phi_{K, k} \Pi_{k} H_{k} H_{k}^{*} \Pi_{k} \Phi_{K}^{*}, k \quad \text { for all } k \geq n
$$

is given by

$$
\operatorname{rank} \sum_{k=0}^{K-1} \theta_{0, k} \psi_{k} G_{k} G_{k}^{*} \psi_{k}^{\theta} 0, k=n \text { for all } k \geq n
$$

where $\psi_{k}$ is defined by (4.7) and $\theta_{0, k}$ is the transition matrix of the (adjoint) system $q_{k}=F_{k+1}^{*} q_{k+1}$. Since $\theta_{0, k}=F_{1}^{*} F_{2}^{*} \ldots F_{k}^{*}$, we have $\theta_{0, k}=\Phi_{k+1,0}^{*}$, yielding the regulability condition

$$
\operatorname{rank} \sum_{k=0}^{K-1} \Phi_{k+1,0}^{*} \psi_{k} G_{k} G_{k}^{*} \psi_{k} \Phi_{k+1,0}=n \text { for all } K \geq n
$$

which is the same as the condition obtained in section 3 . The continuous-time case can be treated in a similar manner.

\section{Conclusion}

The estimability and the regulability properties of linear systems determine the ability to reduce the mean square error in state estimation and the quadratic cost in state feedback regulation strictly below their values when no observations or feedback are used. These properties, which are different from the well known observability and controllability properties, have been shown to be dual. Necessary and sufficient conditions for estimability and for regulability have been derived for time variant and for time invariant systems in continuous and discrete time. 
$\underline{\text { References }}$

[1] R. E. Kalman, "On the General Theory of Control Systems," Proceedings of the First International Congress on Automatic Control, Butterworth's, London, pp. 481-493, 1960.

[2] Y. Baram and U. Shaked, "Minimal Order Estimation of Multivariable Discrete-Time Stochastic Linear Systems," SIAM J. on Control and Optimization, vol. 24, no. 4, pp. 817-820, July 1986.

[3] Y. Baram and U. Shaked, "Minimal Order Estimation of Multivariable Continuous-Time Stochastic Linear Systems," IEEE Trans. on Automatic Control, vol. AC-30, no. 5, pp. 483-484, May 1985.

[4] H-E Chen, Recursive Estimation and Control for Stochastic Systems, Wiley, New York, 1985.

[5] A. E. Bryson, Jr., "Kalman Filter Divergence and Aircraft Motion Estimators," AIAA J. of Guidance and Control, vol. 1, no. 1, pp. 71-79, January 1978.

[6] B. D. O. Anderson and J. B. Moore, Optimal Filtering, Prentice-Hall, Englewood Cliffs, New Jersey, 1979.

[7] T. Kailath, Linear Systems, Prentice-Hall, Englewood Cliffs, New Jersey, 1979. 
[8] P. I. Faurre, "Stochastic Realization Algorithms," in System Identification, R. K. Mehra and D. G. Lainiotis, eds., Academic Press, New York, 1976.

[9] Y. Baram and U. Shaked, "Order Minimality of Multivariable, ContinuousTime Stochastic Realizations," Proceedings of the Seventh IFAC Symposium on Identification and System Parameter Estimation, pp. 765-768, York, United Kingdom, July 1985.

[10] T. Kailath, "Some Chandrasekhar-Type Algorithms for Quadratic Regulators," Proceedings of the Conference of Decision and Control, pp. 219-223, New Orleans, December 1972.

[11] H. Kwakernaak and R. Sivan, Linear Optimal Control Systems, WileyInterscience, New York, 1972. 


\begin{tabular}{|c|c|c|c|}
\hline \multicolumn{4}{|c|}{ Report Documentation Page } \\
\hline $\begin{array}{l}\text { 1. Report No. } \\
\text { NASA TM-89427 }\end{array}$ & 2. Government Accession No. & \multicolumn{2}{|c|}{ 3. Recipient's Catalog No. } \\
\hline \multicolumn{2}{|l|}{ 4. Title and Subtitle } & $\begin{array}{l}\text { 5. Report Date } \\
\text { February } 1\end{array}$ & \\
\hline \multicolumn{2}{|c|}{ Estimability and Regulability of Linear Systems } & \multicolumn{2}{|c|}{ 6. Performing Organization Code } \\
\hline \multirow{2}{*}{\multicolumn{2}{|c|}{$\begin{array}{l}\text { 7. Author(s) } \\
\text { Yoram Baram and Thomas Kailath (Stanford } \\
\text { University, Stanford, CA 94305) }\end{array}$}} & \multicolumn{2}{|c|}{$\begin{array}{l}\text { 8. Performing Organization Report No. } \\
\text { A-87100 }\end{array}$} \\
\hline & & \multicolumn{2}{|c|}{$\begin{array}{l}\text { 10. Work Unit No. } \\
505-66-11\end{array}$} \\
\hline \multirow{2}{*}{\multicolumn{2}{|c|}{$\begin{array}{l}\text { 9. Performing Organization Name and Address } \\
\text { Ames Research Center } \\
\text { Moffett Field, CA } 94035\end{array}$}} & \multicolumn{2}{|c|}{ 11. Contract or Grant No. } \\
\hline & & \multirow{2}{*}{\multicolumn{2}{|c|}{$\begin{array}{l}\text { 13. Type of Report and Period Covered } \\
\text { Technical Memorandum }\end{array}$}} \\
\hline \multirow{2}{*}{\multicolumn{2}{|c|}{$\begin{array}{l}\text { 12. Sponsoring Agency Name and Address } \\
\text { National Aeronautics and Space Administration } \\
\text { Washington, DC } 20546\end{array}$}} & & \\
\hline & & \multicolumn{2}{|c|}{ 14. Sponsoring Agency Code } \\
\hline \multicolumn{4}{|c|}{$\begin{array}{l}\text { Point of Contact: } \begin{array}{l}\text { Yoram Baram, Ames Research Center, M/S 210-9 } \\
\text { Moffett Field, CA } 94035 \text { (415) } 694-5433 \text { or FTS 464-5433 }\end{array}\end{array}$} \\
\hline \multicolumn{4}{|c|}{$\begin{array}{l}\text { A linear state-space system will be said to be estimable if in estimating } \\
\text { its state from its output the posterior error covariance matrix is strictly } \\
\text { smaller than the prior covariance matrix. It will be said to be regulable if } \\
\text { the quadratic cost of state feedback control is strictly smaller than the cost } \\
\text { when no feedback is used. These properties, which are shown to be dual, are } \\
\text { different from the well known observability and controllability properties of } \\
\text { linear systems. Necessary and sufficient conditions for estimability and regul- } \\
\text { ability are derived for time variant and time invariant systems, in discrete and } \\
\text { continuous time. }\end{array}$} \\
\hline $\begin{array}{l}\text { 17. Key Words ISuggested by Auth } \\
\text { Estimation } \\
\text { Regulation } \\
\text { Feedback control } \\
\text { Order minimality }\end{array}$ & $\begin{array}{l}\text { 18. Distril } \\
\text { Uncla }\end{array}$ & -Unlimited & 66 \\
\hline $\begin{array}{l}\text { 19. Security Classif. (of this report) } \\
\text { Unclassified }\end{array}$ & $\begin{array}{l}\text { 20. Security Classif. (of this page) } \\
\text { Unclassified }\end{array}$ & $\begin{array}{c}\text { 21. No. of pages } \\
31\end{array}$ & 22. Price \\
\hline
\end{tabular}

